\title{
Application of Moments Method to Dynamics of Muon Cooling System
}

\author{
Zohreh Parsa ${ }^{\dagger 1}$ and Pavel Zenkevich ${ }^{\ddagger}$ \\ † Department of Physics, Bldg. $901 \mathrm{~A}$ \\ Brookhaven National Laboratory \\ PO Box 5000 Upton, NY 11973-5000 \\ Institute for Theoretical and Experimental Physics \\ $B$ Cheremushkinskaya Ulitsa 25, RU-117 259, Moscow, Russia
}

\begin{abstract}
Application of moments method to study dynamics of muon cooling system is presented. The muon cooling channel has specific features such as damping due to ionization cooling, heating due to multiple scattering and energy struggling. Our method (in a case of transverse motion) is correct in absence of longitudinal magnetic fields such as solenoids.
\end{abstract}

\section{INTRODUCTION}

It is well known that moments method is successfully used for calculation of the dynamics in different focusing channels. However, the muon cooling channel has some specific features: a) damping due to ionization cooling; b) heating due to multiple Coulomb scattering and energy struggling. In our paper [1] on "Kinetics of Muon Longitudinal Cooling" we considered an evolution of longitudinal beam moments in a system with constant parameters. In this paper we investigate a more general case: a cooling system, consisting of a number of sections, each of which has constant parameters. Our method (in a case of transverse motion) is correct in absence of longitudinal magnetic field (e.g. solenoids).

1) Supported by US Department of Energy Contract No. DE-AC02-76CH00016 and National Science Foundation NSF-PHY-94-07194

(C) 1997 American Institute of Physics 


\section{METHOD OF MOMENTS}

Equation of motion for individual particle in presence of damping and random heating may be written as:

$$
y^{\prime \prime}+2 \alpha y^{\prime}+w^{2} y=W(z)
$$

The $y$ is a particle coordinate (transverse or longitudinal one), $2 \alpha$ is a damping coefficient, $w$ is a gradient of the external field, $W(z)$ is a function describing heating.

We can consider $W(z)$ as a sum of random $\delta$-functions (kicks), with given spectral density:

$$
\frac{d\left\langle y^{\prime 2}\right\rangle}{d z}=W
$$

with $y^{\prime}=d y / d z$. Let us assume that $W$ is also constant. Then, by simple differentiation we find (using Eq. 1), that

$$
\left.\begin{array}{rl}
\frac{d\left\langle y^{2}\right\rangle}{d z} & =\left\langle y y^{\prime}\right\rangle \\
\frac{d\left\langle y y^{\prime}\right\rangle}{d z} & =\left\langle\left(y^{\prime}\right)^{2}\right\rangle-2 \alpha\left\langle y y^{\prime}\right\rangle-\Omega^{2}\left\langle y^{2}\right\rangle \\
\frac{d}{d z}\left\langle\left(y^{\prime}\right)^{2}\right\rangle & =4 \alpha\left\langle\left(y^{\prime}\right)^{2}\right\rangle+2 \Omega^{2}\left\langle y y^{\prime}\right\rangle+W
\end{array}\right\}
$$

Let $\left\langle y^{2}\right\rangle=u ;\left\langle y y^{\prime}\right\rangle=v ;\left\langle\left(y^{\prime}\right)^{2}\right\rangle=t$. We can rewrite our system in the following form

$$
\left.\begin{array}{l}
u^{\prime}-2 v=0 \\
\Omega^{2} u+v^{\prime}+2 \alpha v-t=0 \\
-2 \Omega^{2} v+t^{\prime}+4 \alpha t=W
\end{array}\right\}
$$

This system should be solved for general initial conditions:

$$
u=u_{0} ; \quad v=v_{0} ; \quad t=t_{0} .
$$

The solution of the linear system (4) can be written in matrix form:

$$
\left(\begin{array}{c}
u_{f} \\
v_{f} \\
t_{f}
\end{array}\right)=W(R)+\left(\begin{array}{c}
M \\
\end{array}\right)\left(\begin{array}{c}
u_{0} \\
v_{0} \\
t_{0}
\end{array}\right)
$$

Here subscript " $f$ " corresponds to final point of the section, $(R)$ is a column and $(M)$ is $3 \times 3$ matrix.

Elements of $R$ and $M$ may be found using Laplace transform method. We have the following steps: 
1. Using Laplace transform method we get a system of linear equations for $U(p) ; V(p) ; T(p) \rightarrow$ Laplace transforms of $u(z) ; v(z) ; t(z) ;(p$ is a Laplace variable).

2. Solving this linear system we obtain expressions for $U(p) ; V(p) ; T(p)$.

3. A backward Laplace transform can be found using a residue theorem:

$$
f(z)=\sum_{k} \operatorname{res} F\left(p_{k}\right) \exp \left(p_{k} z^{\prime}\right)
$$

Here summation is made over all $p_{k}$ which are the first order residues of function $F(p)$.

Using this procedure, we can obtain final expressions for all the elements.

$$
\begin{aligned}
M_{1,1}= & \exp (-2 \alpha z)\left[\operatorname{ch} 2 \alpha_{0} z+\frac{\alpha}{\alpha_{0}} \operatorname{sh} 2 \alpha_{0} z+\right. \\
& \left.\frac{\Omega^{2}}{2 \alpha_{0}^{2}}\left(\operatorname{ch} 2 \alpha_{0} z-1\right)\right] \\
M_{1,2}= & \exp (-2 \alpha z)\left[\frac{\alpha}{\alpha_{0}^{2}}\left(\operatorname{ch} 2 \alpha_{0} z-1\right)+\frac{s h 2 \alpha_{0} z}{\alpha_{0}}\right] \\
M_{1,3}= & \exp (-2 \alpha z) \frac{\operatorname{ch} 2 \alpha_{0} z-1}{2 \alpha_{0}^{2}} \\
M_{2,1}= & \exp (-2 \alpha z)\left[\frac{\Omega^{2} \alpha}{2 \alpha_{0}^{2}}\left(1-\operatorname{ch} 2 \alpha_{0} z\right)-\frac{\Omega^{2}}{2 \alpha_{0}} \operatorname{sh} 2 \alpha_{0} z\right] \\
M_{2,2}= & \exp (-2 \alpha z)\left[\alpha\left(\operatorname{ch} 2 \alpha_{0} z-1\right)+\alpha_{0} \operatorname{sh} 2 \alpha_{0} z\right] \\
M_{2,3}= & \exp (-2 \alpha z)\left[\frac{\alpha}{2 \alpha_{0}^{2}}\left(1-\operatorname{ch} 2 \alpha_{0} z\right)+\frac{\operatorname{sh} 2 \alpha_{0} z}{2 \alpha_{0}}\right] \\
M_{3,1}= & \exp (-2 \alpha z)\left[\frac{\Omega^{4}}{2 \alpha_{0}^{2}}\left(\operatorname{ch} 2 \alpha_{0} z-1\right)\right] \\
M_{3,2}= & \exp (-2 \alpha z) \Omega^{2}\left[\frac{\alpha}{\alpha_{0}^{2}}\left(1-\operatorname{ch} 2 \alpha_{0} z\right)+\frac{\operatorname{sh} 2 \alpha_{0} z}{\alpha_{0}}\right] \\
M_{3,3}= & \exp (-2 \alpha z)\left[\frac{\Omega^{2}}{2 \alpha_{0}^{2}}\left(\operatorname{ch} 2 \alpha_{0} z-1\right)+\left(\operatorname{ch} 2 \alpha_{0} z\right.\right. \\
& \left.\left.\quad-\frac{\alpha}{\alpha_{0}} \operatorname{sh} 2 \alpha_{0} z\right)\right]
\end{aligned}
$$

All diagonal terms $\left(M_{1,1}, M_{2,2}\right.$, and $\left.M_{3,3}\right)$ should be equal to 1 for $z=0$, all others are equal to 0 for $z=0$. For column:

$$
R_{1}=\frac{1}{4 \alpha \Omega^{2}}\left[1+\frac{\Omega^{2}}{\alpha_{0}^{2}} \exp (-2 \alpha z)-\frac{\alpha \exp (-2 \alpha z)}{\alpha_{0}^{2}}\right.
$$




$$
\begin{gathered}
\left.\left(\alpha_{0} \operatorname{sh} 2 \alpha_{0} z+\alpha \operatorname{ch} 2 \alpha_{0} z\right)\right] \\
R_{2}=\frac{\exp (-\alpha z)}{4 \alpha_{0}^{2}}\left(\operatorname{ch} 2 \alpha_{0} z-1\right) \\
R_{3}=\frac{1}{4 \alpha \Omega}\left\{1+\exp (-2 \alpha z)\left[\operatorname{sh} 2 \alpha_{0}\left(1-\frac{\alpha}{\alpha_{0}}\right)-\frac{\alpha^{2} \operatorname{ch} 2 \alpha_{0} z-\Omega^{2}}{\alpha_{0}^{2}}\right]\right\}
\end{gathered}
$$

Here

$$
\alpha_{0}=\sqrt{\alpha^{2}-\Omega^{2}}
$$

If $\alpha_{0}$ is imaginary, then

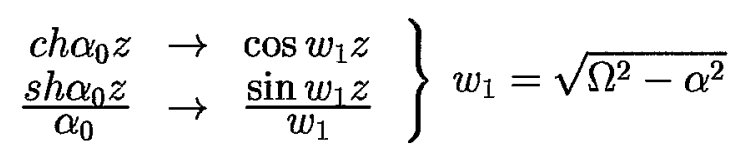

\section{APPLICATIONS TO LONGITUDINAL COOLING}

In this case $y=z-z_{s}$. $\left(z_{s}\right.$ is a coordinate of equilibrium particle),

$$
\begin{array}{r}
2 \alpha=\frac{1}{m c^{2} \beta^{2} \gamma}\left(-\frac{d E}{d z}\right)_{\text {ion }} \frac{\Psi}{L_{0}}+\frac{d}{d z}\left(\frac{p}{\Lambda}\right) / \frac{p}{\Lambda}- \\
\frac{d}{d p}\left[\left(\frac{d E}{d z}\right)_{\text {ion }} \frac{1}{v}\right]=2 \alpha_{1}+2 \alpha_{2}+2 \alpha_{3}
\end{array}
$$

The first term in RHS describes longitudinal cooling due to wedges, the second one damping due to change of longitudinal mass, and the third one - so named "natural" cooling due to slope of the ionization losses curve [2]. Here

$$
L_{0}=\frac{\left(\frac{d E}{d z}\right)_{\text {ion }}}{\frac{d}{d X}\left(\frac{d E}{d z}\right)_{\text {ion }}}
$$

We see, that if $\left(\frac{d E}{d z}\right)_{\text {ion }}$ does not depend on $X$, the derivative is equal to zero, and the first term in (22) disappears. Where $\mathrm{X}$ is a transverse coordinate.

Parameter $\Lambda$ is defined by

$$
\Lambda=\left\langle\frac{\Psi}{R}\right\rangle+\frac{1}{\gamma^{2}}
$$

$R$ is a radius of curvature for ideal trajectory. $\Omega$ is the synchrotron frequency and in linear approximation may be written as

$$
\Omega^{2}=\frac{2 \pi E_{a c} \cos \left(\frac{2 \pi}{\lambda} z_{s}\right) \Lambda}{m c^{2} \lambda \beta^{2} \gamma}
$$


Here $E_{a c}$ is an amplitude of r.f. field, and $\lambda$ is its wavelength.

$$
\begin{gathered}
W_{y}=\left(\frac{p}{\Lambda}\right)^{-2} \frac{1}{\beta^{2} m c^{2}} \frac{d}{d z}\left\langle(\Delta E)^{2}\right\rangle \\
\frac{d}{d z}\left\langle(\Delta E)^{2}\right\rangle=K_{s} \gamma^{2}\left(1-\frac{\beta^{2}}{2}\right) \\
K_{s}=4 \pi\left(r_{e} m_{e} c^{2}\right)^{2} \frac{\rho Z}{A}
\end{gathered}
$$

Here $r_{e}, m_{e}$ - classical radius and mass of electron; $\rho ; Z ; A$ are density; charge and atomic number of the wedge material $m$ - muon mass. Let us consider sections which are specific for cooling system:

a) Wedge section

$$
\alpha=\alpha_{1}+\alpha_{2}+\alpha_{3} ; W=W_{y} ; \Omega_{s}^{2}=0
$$

b) Section of transverse cooling $(\Psi=0)$

$$
\alpha=\alpha_{2}+\alpha_{3} ; W+W_{y} ; \Omega_{s}^{2}=0
$$

c) Accelerating section

$$
\alpha=\alpha_{2} ; W=0 ; \Omega_{s}^{2} \neq 0
$$

\section{APPLICATIONS TO TRANSVERSE COOLING}

Here $y$ is a transverse coordinate

$$
\alpha=-\left(\frac{d E}{d z}\right)_{\text {ion }} \frac{1}{m c^{2} \beta^{2} \gamma}-K \alpha_{1}+\frac{p^{\prime}}{p}
$$

$K$ is a coefficient describing coupling with "wedge section", if $\Psi=\Psi_{y}, K=1$; if $\Psi_{y}=0, K=0$.

$$
W=W_{\perp}=\frac{E_{s}^{2}}{(p c \beta)^{2}} \frac{1}{X_{r}}
$$

$E_{s}=15 \mathrm{MeV}, X_{r}$ is material radiation length. Let us consider sections which are specific for ionization cooling system.

a) Wedge section

$W=W_{\perp}$

$\alpha=\alpha_{\perp}^{0}-K \alpha_{1}+\frac{p^{\prime}}{p}$

It is easy to show, that $\frac{p^{\prime}}{p}+\alpha_{\perp}^{0}=0$,

$\Omega^{2}$ is arbitrary 
b) Section of transverse cooling

$W=W_{\perp}$

$\alpha=0$

$\Omega^{2}$ is arbitrary

c) Accelerating section

$W=0$

$\alpha=\frac{p^{\prime}}{p}$

$\Omega^{2}$ is usually zero.

Since in our variables $X, \frac{d X}{d z}$; damping rate (in absence of wedges) is equal to zero, if wedges are used, then we have increment $(\alpha<0)$ in such a section.

\section{ACKNOWLEDGEMENTS}

This work was partially carried out at the Institute for Theoretical Physics, University of California, Santa Barbara, as part of the New Ideas for Particle Accelerators Program with partial support by the the National Science Foundation Grant No. NSF-PHY-94-07194.

\section{REFERENCES}

1. Z. Parsa, P. Zenkovich, Kinetics of Muon Longitudinal Cooling, to be published (1997).

2. V. Parchomchuk and A. Skrinsky, AIP CP 372, 139 (1996). 